\title{
Impactos da utilização de dispositivos contraceptivos reversíveis de longa duração na
}

\section{saúde feminina}

Impacts of the use of long-term reversible contraceptive devices on women's health

Impactos del uso de dispositivos anticonceptivos reversibles a largo plazo en la salud de la mujer

Recebido: 14/11/2021 | Revisado: 27/11/2021 | Aceito: 28/11/2021 | Publicado: 01/12/2021

Elizabeth Carvalho da Silva

ORCID: https://orcid.org/0000-0003-1533-2879

Universidade Nilton Lins, Brasil E-mail: ecarv.96@gmail.com

Ronaldo da Silva Ferreira

ORCID: https://orcid.org/0000-0002-1966-8081

Universidade de Aveiro, Portugal

E-mail: ronaldoferreira@ua.pt

Lucilene Ferreira Mouzinho

ORCID: https://orcid.org/0000-0002-9825-6009

Instituto Federal do Maranhão, Brasil E-mail: mouzinho@ifma.edu.br

Vitor Hugo Paixão Ferreira

ORCID: https://orcid.org/0000-0001-7537-6596

Universidade de Aveiro, Portugal

E-mail: vferreira@av.it.pt

Erick Frota Gomes Figueiredo

ORCID: https://orcid.org/0000-0002-6127-0544

Universidade Nilton Lins, Brasil

E-mail: erick.figueiredo@uniniltonlins.edu.br

\begin{abstract}
Resumo
O objetivo deste estudo foi realizar uma revisão sistemática prisma utilizando a técnica prisma que permitiu identificar quais os principais problemas de saúde causados em mulheres que utilizaram dispositivos contraceptivos reversíveis de longa duração. Na metodologia adotada selecionaram-se os artigos por um processo de 04 níveis hierárquicos, utilizando-se critérios de corte. No primeiro nível foram selecionados 1391 artigos publicados entre os anos de 2017 a 2021, nas bases de dados SciELO, Google Acadêmico, PubMed/MEDLINE e Biblioteca Virtual de Saúde BVS/LILACS, utilizando-se palavras chaves como critério. Nos níveis seguintes, selecionaram-se artigos que continham temas, títulos e resumos relacionados aos do trabalho. Após seleção e triagem houve uma redução na quantidade de artigos para 50 selecionados, representando 3,59\% do total, sendo a PubMed/MEDLINE com 40 artigos a plataforma com maior participação correspondendo a 2,88 \%. As demais plataformas apresentaram os seguintes valores: SciELO 02 artigos $(0,14 \%)$; Google acadêmico e LILACS 04 artigos cada $(0,28 \%)$. Identificou-se ainda, os principais problemas de saúde e as incidências nas plataformas: Sangramento (PubMed/MEDLINE 20\%; LILACS e Google acadêmico 4\%); ganho de peso (PubMed/MEDLINE 16\%; BVS/LILACS 4\%); migração do implante de subdérmico (PubMed 28\%); influência na função sexual (PubMed/MEDLINE 6\%); outros (PubMed/MEDLINE 28\%; BVS/LILACS 14\%). Verificou-se, que os principais problemas de saúde relatados foram sangramento e migração do implante. Logo, esta revisão os principais tipos de problemas de saúde causados pelos dispositivos contraceptivos reversíveis de longa duração, num intervalo de tempo curto, não comprometendo a acurácia dos resultados.
\end{abstract}

Palavras-chave: Contraceptivos; Risco à saúde; Intrauterino; DIU; Implanon.

\begin{abstract}
The goal of this study was to carry out a systematic prism review using the prism technique that allowed us to identify the main health problems caused in women who used long-term reversible contraceptive devices. In the adopted methodology, the articles were selected through a process of 04 hierarchical levels, using cutoff criteria. At the first level, 1391 articles published between 2017 and 2021 were selected, in the SciELO, Academic Google, PubMed/MEDLINE and Virtual Health Library (Biblioteca Virtual de Saúde-BVS)/LILACS databases, using keywords as criteria. At the following levels, articles that contained themes, titles and abstracts related to the work were selected. After selection and screening, there was a reduction in the number of articles to 50 selected, representing $3.59 \%$ of the total, with PubMed/MEDLINE with 40 articles being the platform with the highest participation, corresponding to $2.88 \%$. The other platforms presented the following values: SciELO 02 articles (0.14\%); Academic Google and LILACS
\end{abstract}


04 articles each $(0.28 \%)$. The main health problems and incidences in the platforms were also identified: bleeding (PubMed/MEDLINE 20\%; LILACS and Google academic 4\%); weight gain (PubMed/MEDLINE 16\%; BVS/LILACS 4\%); subdermal implant migration (PubMed 28\%); influence on sexual function (PubMed/MEDLINE 6\%); others (PubMed/MEDLINE 28\%; BVS/LILACS 14\%). It was found that the main health problems reported were bleeding and implant migration. Therefore, this reviews the main types of health problems caused by reversible contraceptive devices of long duration, in a short period of time, without compromising the accuracy of the results.

Keywords: Contraceptives; Health risk; Intrauterine; IUD; Implan.

\section{Resumen}

El objetivo de este estudio fue realizar una revisión sistemática de la literatura mediante la técnica del prisma, que permitió identificar los principales problemas de salud que se producen en las mujeres que utilizan anticonceptivos reversibles de larga duración. En la metodología adoptada, los artículos fueron seleccionados mediante un proceso de 04 niveles jerárquicos, utilizando criterios de corte. En un primer nivel, se seleccionaron 1391 artículos publicados entre 2017 y 2021, en las bases de datos SciELO, Google académico, PubMed/MEDLINE y librería virtual de salud (Biblioteca Virtual de Saúde-BVS)/LILACS, utilizando como criterio palabras clave. En los siguientes niveles se seleccionaron artículos que contenían temas, títulos y resúmenes relacionados con el trabajo. Tras la selección y cribado, se redujo el número de artículos a 50 seleccionados, lo que representa el 3,59\% del total, siendo PubMed/MEDLINE con 40 artículos la plataforma con mayor participación, correspondiente al 2,88\%. Las demás plataformas presentaron los siguientes valores: artículos SciELO 02 (0,14\%); Artículos académicos de Google y LILACS 04 cada uno (0,28\%). También se identificaron los principales problemas e incidencias de salud en las plataformas: Sangrado (PubMed/MEDLINE 20\%; LILACS y Google académico 4\%); Aumento de peso (PubMed/MEDLINE 16\%; BVS/LILACS 4\%); migración subdérmica del implante (PubMed 28\%); influencia en la función sexual (PubMed/MEDLINE 6\%); otros (PubMed/MEDLINE 28\%; BVS/LILACS 14\%). Se encontró que los principales problemas de salud reportados fueron el sangrado y la migración de los implantes. Por ello, en esta revisión se revisan los principales tipos de problemas de salud provocados por los dispositivos anticonceptivos reversibles de larga duración, en un período corto de tiempo, sin comprometer la exactitud de los resultados.

Palabras clave: Anticonceptivos; Riesgo de salud; Intrauterino; DIU; Implanon.

\section{Introdução}

Nas últimas décadas, as mulheres vêm ocupando cada vez mais posições de destaque em vários setores da sociedade e economia em todo mundo, como indústria, comércios, tecnologia e educação (Visentini, 2021). Esses espaços, majoritariamente, antes ocupados por homens nos últimos anos vêm apresentando mudanças significativas nesse cenário (Vasconcellos \& Brizola, 2016). Apesar disso, elas enfrentam mais dificuldades, por exemplo, no setor econômico, em conseguirem empregos que em sua maioria são de baixas qualidades e remunerações (Santos, 2020).

Um dos principais fatores para o aumento do protagonismo feminino no setor econômico é justamente por estarem melhor qualificadas técnico - educacionalmente, que as fazem mais preparadas para o mercado de trabalho (Fleith, Gomes, Araújo \& Almeida, 2020). Isso é evidenciado não somente, mas principalmente pelos números de mulheres nos cursos de graduação e pósgraduação que já ultrapassam os números de homens, em quase todas as universidades do mundo (Milletler, 2020). Isso mostra a preocupação crescente das mulheres no investimento pessoal em suas formações e carreiras profissionais, acarretando melhores colocações profissionais e salários (Rodrigues, Rodrigues, Silva \& Ferreira, 2021).

Dessa forma, uma gravidez não planejada pode comprometer os anseios profissionais de mulheres em início de carreira. Portanto, entra em cena o controle da fecundidade feminina, através do uso de métodos contraceptivos (Couto, Saiani \& Kuwahara, 2020). Eles possuem a finalidade de impedir uma gravidez indesejada ocorrida por meio de um ato sexual, assumindo uma posição de destaque não apenas no controle de natalidade, mas na prevenção de doenças (Silva et al., 2021).

Os métodos contraceptivos são divididos em duas categorias, de acordo com a maneira que agem no organismo feminino: métodos contraceptivos reversíveis e irreversíveis. Os métodos contraceptivos reversíveis são aqueles que podem ser interrompidos a qualquer momento sem prejuízo à saúde feminina e quanto aos seus tipos são: os comportamentais; métodos de barreira; dispositivos intrauterinos de cobre e hormonais, e métodos de emergência. Os métodos contraceptivos irreversíveis são 
aqueles que são em geral realizados por via cirúrgica e que causa esterilidade permanente. Quanto aos tipos eles podem ser definidos como: esterilização cirúrgica feminina e masculina (Junges at al, 2021).

Nos países desenvolvidos estima-se que 70\% das mulheres casadas (ou em união estável) utilizem ou já utilizaram algum método a fim de evitar uma gravidez, e 62\% nos países subdesenvolvidos (Mendes, 2021). Ainda nos países subdesenvolvidos, os números relativos ao uso de técnicas contraceptivas mais modernas ficaram na faixa de $56 \%$ da população feminina sexualmente ativa (Bearak, Popinchalk, Alkema \& Sedgh, 2018). Já com relação a outras técnicas, como esterilização e uso de dispositivos intrauterinos o percentual é menor, porém significativo ficando com 22\% e 15\%, respectivamente (Mendes, 2021). Estes índices são marcantes devido aos seus valores elevados, e demonstram como ocorre a distribuição dessas técnicas de contracepção mais modernas nas camadas sociais, de acordo com variáveis educacionais e econômicas. O uso dos LARC's (Long-Acting Reversible Contraceptives), isto é, dos métodos contraceptivos reversíveis de longa duração pela população feminina nesses países, vem incidindo nos últimos anos, na redução da fecundidade dessa população (Brandão, 2019).

Apesar de alcançarem suas expectativas de reduzirem as taxas de natalidade, os LARC's apresentam atrelados a isso, alguns problemas aos seus usos como alterações na microbiota vaginal (Donders, Bellen, Ruban \& Bulck; Achille, 2018), (Bassis et al., 2017); gravidez ectópica (Simon, Smith \& Holzhauer, 2017); efeitos colaterais androgênicos dermatológicos (Lullo et al., 2017), abscesso pélvico causado por perfuração do colón sigmóide (Toumi et al., 2017), perfuração uterina (O’Brien \& Pillai, 2017), risco de câncer de mama (Mørch et al., 2017), sangramento uterino anormal (Huss, Schaap \& Kromhout, 2017), (Cim et al., 2017), (Carvalho et al., 2017), fator de risco para tratamento cirúrgico com portadores de abscesso tubo-ovário (TOA) (Inal, Inal \& Gorkem, 2017), abscesso pélvico (Boisen, Ostenfeldt, Erikstrup \& Bay, 2017), depressão, disfunção sexual, distúrbios menstruais (Madliki, Hofmeyr, d'Hellencourt \& Lawrie, 2017), retenção do dispositivo intrauterino, expulsões reconhecidas (Colwill, Schreiber, Sammel \& Sonalkar, 2017), risco de câncer cervical (Cortessis et al., 2017), migração do dispositivo intrauterino em bexiga com formação de pedra (Silva, Kodithuwakku, Aponsu, Rathnayake \& Rajasegaram, 2017), (Sano, Nemoto, Miura \& Suzuki, 2017), migração do dispositivo causando apendicite aguda (Parlakgumus, Parlakgumus \& Ezer, 2017), translocação intra-abdominal (Uçar, Şanlıkan, Ilhan, Göçmen \& Çelik, 2017), gravidez por uso prolongado (Bahamondes \& Monteiro, 2017), amenorréia (Darney et al., 2017), Trichomonas vaginalis (Santos, Rigo, Macedo \& Tasca, 2017), Chlamydia (Rose, Garrett, Stanley \& Pullon, 2017), transmigração vesical (Chai, Zhang, Jia, Cui \& Cui, 2017), (Clancy, Gerritzen \& Pascali, 2017), (Sharma, Andankar \& Pathak, 2017), possível toxicidade sistêmica crônica (Zhao, Liu, Sun, Hu \& Wang, 2017), actinomicose genital (Boisen, Ostenfeldt, Erikstrup \& Bay, 2017), actinomicose abdominal e pélvica (Nakashira, Maximiano, Lima \& Ussami, 2017), dentre outros.

Entre esses vários problemas de saúde registrados na literatura, este trabalho se propõe a realizar uma revisão sistemática de literatura utilizando a técnica prisma, que permitiu indicar quais foram os principais problemas de saúde causados em mulheres que utilizaram dispositivos contraceptivos reversíveis de longa duração, através da análise de periódicos das bases de dados como: SciELO, Google Acadêmico, PubMed/MEDLINE e BSV/LILACS (Ti et al., 2021). Isso é possível, devido à capacidade de uma revisão sistemática da literatura, possibilitar responder a uma ou mais perguntas a partir de estudos primários (Paul, \& Criado, 2020). O processo metodológico usado consistiu-se numa seleção hierárquica composta de 04 níveis. Os critérios de corte para selecionar os artigos encontrados foram definidos a partir da identificação de palavras-chaves, tema, título e resumos relacionados com o tema abordado. Essa metodologia permitiu identificar mulheres em idades férteis, juntamente com os problemas de saúde causados pelo uso de dispositivos contraceptivos reversíveis de longa duração, como maior incidência em cada base de dados.

Apesar dos artigos selecionados estarem compreendidos entre 2017 a 2021, ser uma faixa de tempo curta, isso não compromete a credibilidade dos dados obtidos. A metodologia aqui apresentada, permite realizar uma revisão sistemática da 
literatura para os problemas de saúde relacionados ao tema nas bases de dados aqui identificadas, que resulta também numa redução no tempo de busca, agilizando a revisão sem comprometer os resultados.

\section{Dispositivos contraceptivos reversíveis de longa duração}

Os dispositivos contraceptivos reversíveis de longa duração são métodos de contracepção reversíveis, caracterizados pelo longo tempo de ação no organismo feminino. Os principais representantes destes são: os dispositivos intrauterinos, de cobre ou hormonais e os implantes subdérmicos.

\subsection{Dispositivos intrauterinos - DIU}

De acordo com Silva et al. (2014), o dispositivo intrauterino - DIU consiste num pequeno aparelho introduzido no útero feminino, cuja finalidade é prevenir uma gestação. Esse procedimento é realizado por um médico e o período de realização desse procedimento é em geral durante o período menstrual. Existem atualmente 02 tipos desses dispositivos, o DIU de cobre e o de ação hormonal. O DIU de cobre é considerado por alguns especialistas em reprodução humana, como sendo o método mais eficaz (Adeyemi-Fowode \& Bercaw-Pratt, 2019). Para outros autores, o dispositivo de ação hormonal é mais eficaz, em virtude de apresentarem melhores resultados em contrapartida aos de cobre, devido ao menor risco de complicação numa descontinuação no seu uso (Dinehart, Lathi \& Aghajanova, 2020).

Na Figura 1, são representados 03 tipos de dispositivos intrauterinos. O primeiro (a), é a versão clássica, pertencente ao modelo DIU de cobre "T", e é o mais utilizado entre dispositivos contraceptivos reversíveis de longa duração, conhecidos no mercado. Tem durabilidade de 10 anos. É destinado a mulheres que buscam um método contraceptivo de longo prazo e não hormonal; o segundo (b), pertence ao modelo DIU de Cobre Andalan Classic Cu 380. Consiste em fácil aplicação médica. Também não é de ação hormonal, e tem vida útil de 10 anos, aproximadamente. E, por fim, (c) o modelo DIU de cobre Andalan Classic $\mathrm{Cu} 380$. Esse modelo possibilita uma melhor adaptação intrauterina e possui menor risco de deslocamento e expulsão, devido a cavidade uterina possuir entre 05 e $09 \mathrm{~cm}$ de diâmetro. É possível ainda, encontrar outras sub variações desse tipo de DIU, no formato da letra grega "ômega".

Figura 1. Dispositivo intrauterinos de cobre: (a) modelo DIU de cobre "T"; (b) DIU de cobre Andalan Classic Cu 380 e (c) DIU de cobre Andalan Classic Cu 380.

(a)

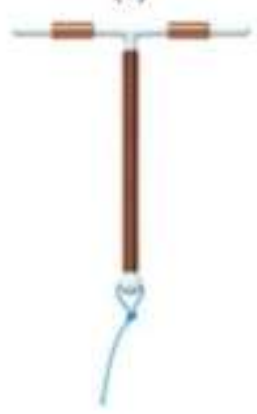

(b)

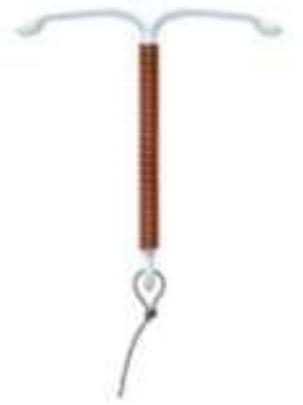

(c)

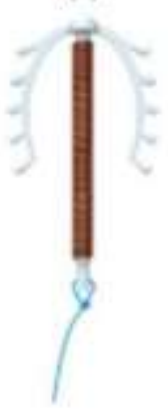

Fonte: Fontenelle (2021).

Os dispositivos intrauterinos hormonais, chamados de DIU de levonorgestrel. Possui uma estrutura de polietileno e são baseados nos modelos Nova T (Albuquerque, 2018). Sua haste é uma junção de polidimetilsiloxane e levonorgestrel moldados em torno do braço vertical, revestida por uma membrana de polidimetilsiloxane. É através dessa membrana que é liberado o hormônio levonorgestrel, que é liberado a uma taxa de $20 \mathrm{mcg}$ de levonorgestrel/dia, por um período médio de 5 anos. Esses 
taxa permite manter a eficácia contraceptiva mesmo em casos de atraso de até 02 na substituição do dispositivo subdérmico, sendo ideal para mulheres que tem o hábito de esquecer-se de tomar as pílulas contraceptivas (Grandi, Farulla, Sileo \& Facchinetti, 2018).

Esse tipo de dispositivo intrauterino, comercialmente recebe o nome de Mirena ${ }^{\circledR}$, e possui um tempo útil de vida de 05 anos. É recomendado ser aplicado após o ciclo menstrual mensal. Esse método é considerado seguro e com alta taxa de prevenção de uma gestação (Adeyemi-Fowode \& Bercaw-Pratt, 2019).

Em relação aos dispositivos intrauterinos de cobre e hormonal, além de suas ações eficazes e duradouras (aproximadamente 5 anos), suas ações no organismo feminino não sofrem influência, pelo comportamento ou estilo de vida das mulheres que o usam. Possuem uma boa relação entre o custo e benefício, e ainda se apresentam com poucos efeitos adversos à saúde. O processo ovulatório, não é inibido, mas no caso do DIU de cobre, pode causar danos aos ovócitos (Giordano, Giordano \& Panisset, 2015), e são nos casos de tumores no útero ou cérvix, hemorragia vaginal, gravidez ou suspeita de gravidez, risco aumentado para a ocorrência gravidez ectópica, presença de IST ou doença inflamatória pélvica, doença de Wilson, ou mulheres alérgicas ao cobre. Existe ainda a tendência de aumento no fluxo menstrual e aparecimento de dores abdominais (AdeyemiFowode \& Bercaw-Pratt, 2019).

\subsection{Dispositivos transdérmicos}

Os dispositivos ou adesivos transdérmicos são contraceptivos constituídos por quatro camadas. A primeira possui um filme de poliéster transparente; A segunda é onde está presente o hormônio é o estradiol (ou o 17ß-estradiol); a terceira camada é formada de uma membrana de copolímero responsável a liberação do fármaco de etileno-coacetato de vinila, e a última camada é a adesiva. Cada adesivo possui uma eficácia de 99,4\%, e contém $750 \mu \mathrm{g}$ de etinilestradiol e 6,0 $\mathrm{mg}(6.000 \mu \mathrm{g})$ de norelgestromina que quando posto sobre a pele libera $20 \mu \mathrm{g}$ de etinilestradiol diariamente. Cerca de $150 \mu \mathrm{g}$ de norelgestromina são absorvidos, e entrando na corrente sanguínea. Na Figura 2, são mostrados (a) adesivos transdérmico; adesivo transdérmico anexado ao antebraço; dorso; ao abdômen; região glútea e na Figura 3, as fórmulas moleculares do estradiol (a) e norelgestromina (b).

Figura 2. Adesivo transdérmico e os locais de sua aplicação no corpo feminino.
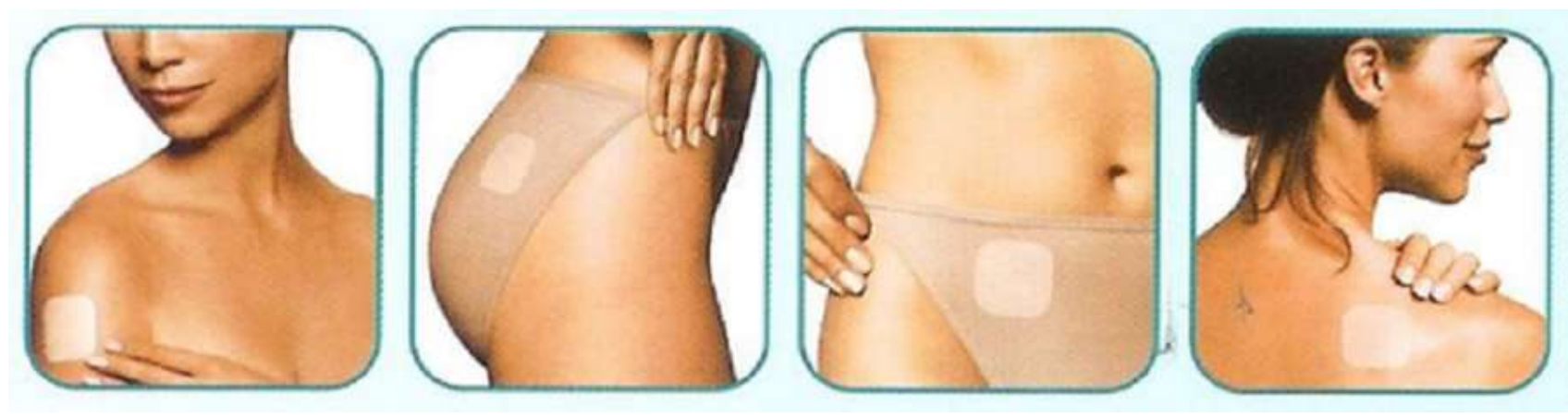

Fonte: Enfermagem Florence, (2020). 
Figura 3. Representação da fórmula molecular de Estradiol (a) e norelgestromina (b), hormônios presentes nos adesivos transdérmicos.

(a)

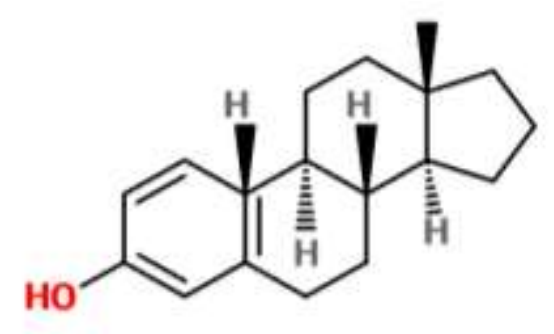

(b)

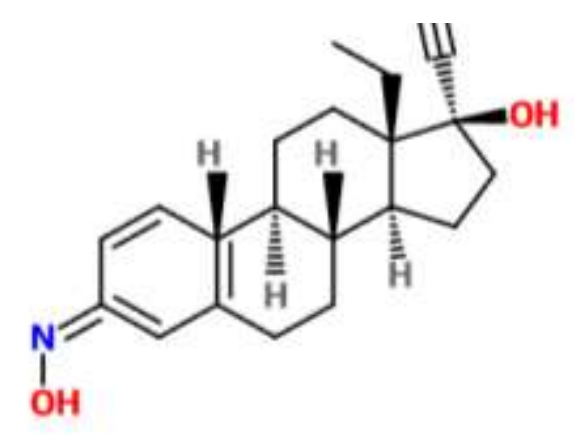

Fonte: Autores (2021).

A principal vantagem associada ao uso dos adesivos contraceptivos transdérmicos é a não oscilações significativas dos níveis de soros-sanguíneos. Quanto a sua aplicação deve ser realizada, semanalmente num período de três semanas, devendo-se suspender o uso no início da quarta semana, para que se tenha a menstruação (Ranieri, 2011, citado por Albuquerque, 2018).

\section{Metodologia}

A metodologia utilizada consistiu em pregar uma revisão sistemática prisma para a realização uma revisão da literatura. O processo foi dividido de forma hierárquica, sendo constituído de 04 níveis, cada um com um critério de corte. No primeiro nível, isto é, o primeiro nível do processo prisma - identificação (a), foram selecionados uma quantidade de 1391 artigos publicados entre os anos de 2017 a 2021 não duplicados, selecionados verificando-se quais tinham as mesmas palavras chaves adotadas para este trabalho. Isso resultou na seguinte distribuição a partir das plataformas de bancos de dados, BVS/LILACS: 902; PubMed/MEDLINE: 367; SciELO: 62 e Google acadêmico: 60 artigos. No segundo nível, isto é, no nível do processo de triagem (b), a seleção ocorreu selecionando-se artigos com temas relacionados com o tema proposto para o trabalho, que gerou a seguinte distribuição, BVS/LILACS: 15; PubMed/MEDLINE: 118; SciELO: 31 e Google acadêmico: 24 artigos. O critério de elegibilidade do prisma, foi dividido da seguinte maneira: terceiro nível, onde a seleção se deu escolhendo-se os artigos com títulos relacionados com o título escolhido para este trabalho que resultou em BVS/LILACS: 15; PubMed/MEDLINE: 113; SciELO: 09 e Google acadêmico: 10 artigos; e, o quarto nível, onde a seleção dos artigos ocorreu selecionando-se aqueles com resumos relacionados com o trabalho proposto, obtendo-se dessa forma BVS/LILACS: 04; PubMed/MEDLINE: 40; SciELO: 02 e Google acadêmico: 04 artigos. Esse esquema pode ser observado na Figura 4, que representa o fluxograma do processo primas, e logo a seguir na Figura 5, que descreve o processo a cada nível hierárquico e com o seu respectivo critério de corte (seleção e exclusão de artigos). 
Research, Society and Development, v. 10, n. 15, e466101523281, 2021

(CC BY 4.0) | ISSN 2525-3409 | DOI: http://dx.doi.org/10.33448/rsd-v10i15.23281

Figura 4. Fluxograma da revisão sistemática prisma

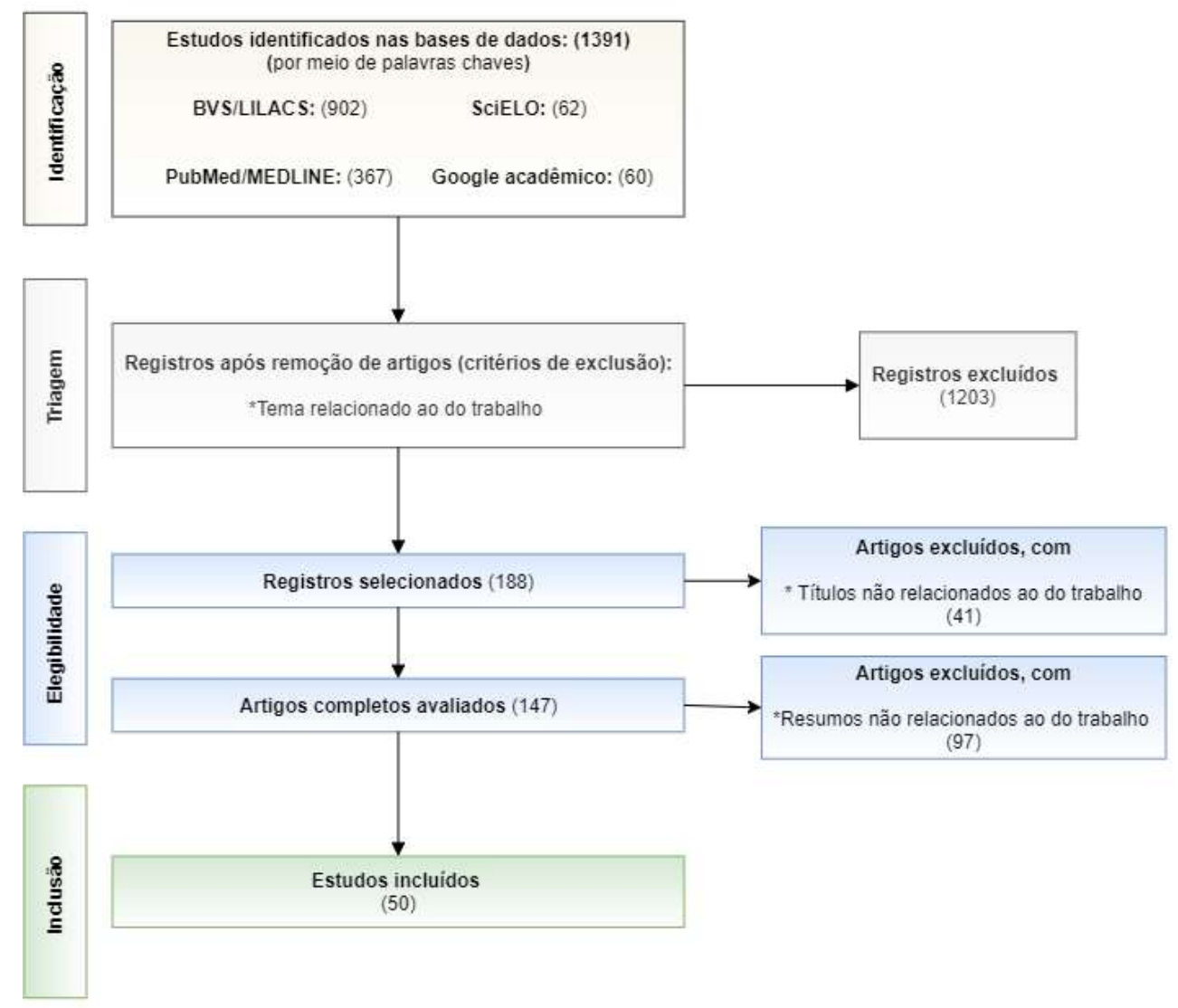

Fonte: Autores (2021). 
Research, Society and Development, v. 10, n. 15, e466101523281, 2021

(CC BY 4.0) | ISSN 2525-3409 | DOI: http://dx.doi.org/10.33448/rsd-v10i15.23281

Figura 5. Seleção hierárquica de artigos utilizados na metodologia para realização da revisão sistemática do trabalho.

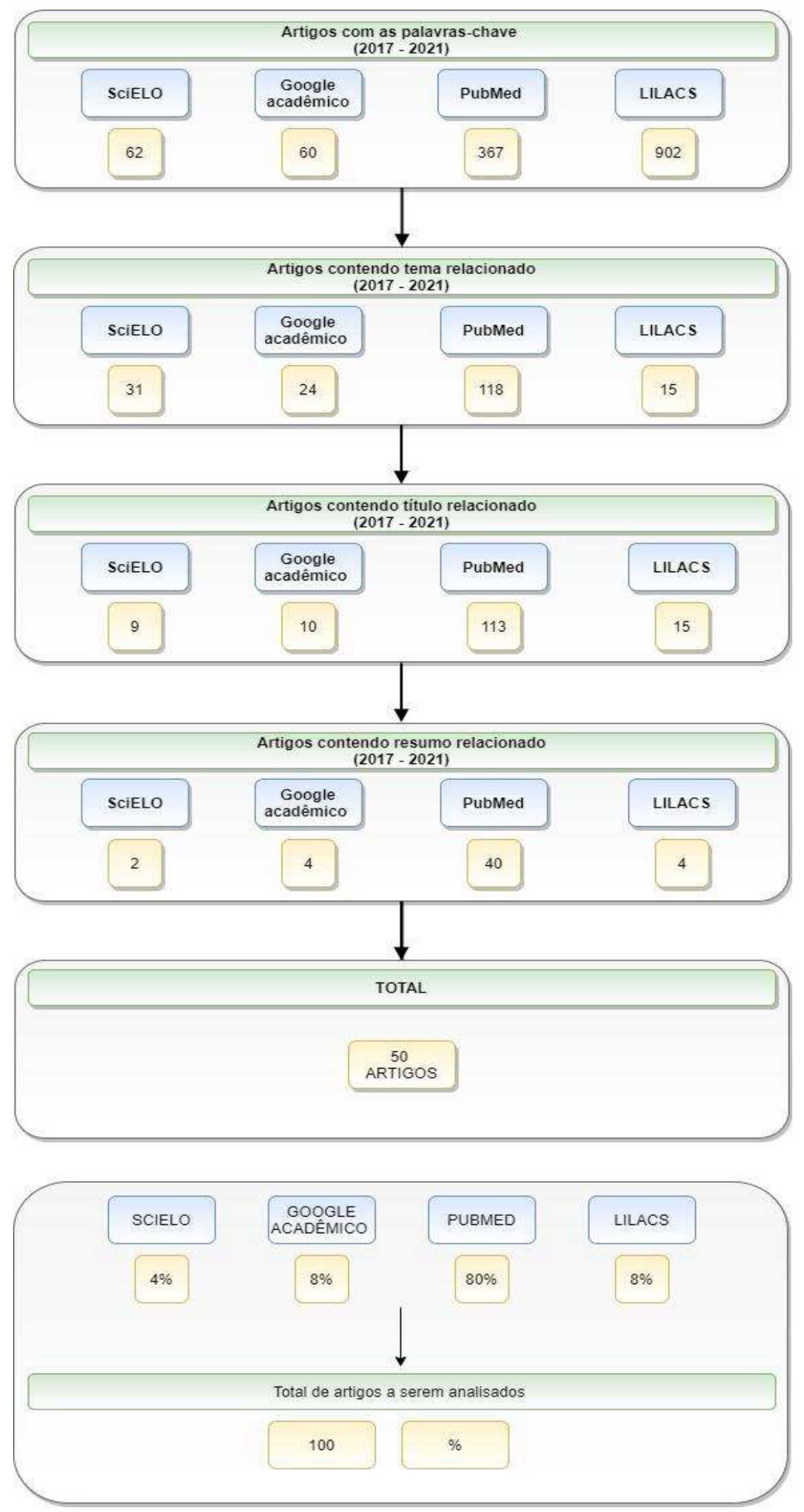

Fonte: Autores (2021). 


\section{Resultados e Discussão}

Os resultados obtidos mostram primeiramente uma redução significativa no número inicial de artigos. O número que inicialmente era de 1391 foi reduzido para 50 artigos selecionados, representando uma redução de 96,40\%. Isso significou que apenas 3,59\% do total de artigos apresentaram importância de acordo com os parâmetros adotados nesta pesquisa. Entre as plataformas de banco de dados, a PubMed/MEDLINE com 40 artigos, apresentou maior participação ficando com a 2,87 \% do total. As demais plataformas apresentaram os seguintes valores: SciELO 02 artigos (0,14\%); Google acadêmico e BVS/LILACS 04 artigos cada (0,28\%). Percentualmente entre as plataformas de dados, a PubMed/MEDLINE se mostrou com $80 \%$ dos artigos selecionados ao final do processo, isto é, no fim do nível 04. As demais ficaram respectivamente com SciELO 4\%; BVS/LILACS e Google acadêmico: $8 \%$. Esse fato pode ser observado no gráfico representado na Figura 6.

Figura 6. Percentual de relevância relativa entre as plataformas de dados, no momento de se realizar buscas sobre os principais problemas de saúde relacionados ao tema da pesquisa.

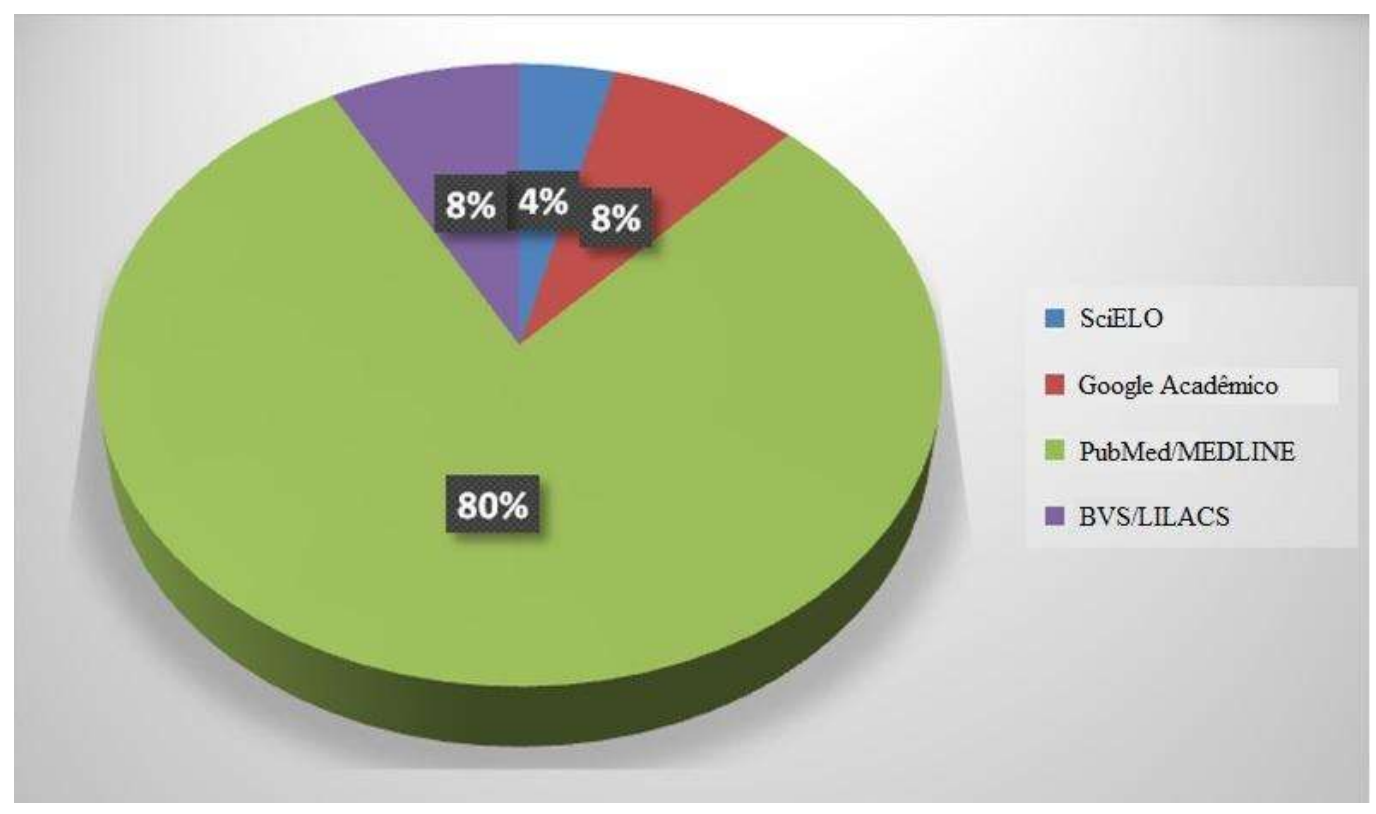

Fonte: Autores (2021).

Como foi observado anteriormente, ao final do processo de seleção dos artigos cerca de $80 \%$ pertencem a plataforma de dados da PubMed/MEDLINE e os $20 \%$ restantes, pertencem as outras 03 plataformas. Foram identificados quais os principais problemas de saúde com maior incidência entre os bancos de dados e os seus respectivos valores percentuais em relação ao número final de 50 artigos selecionados. E, eles foram: sangramento (PubMed/MEDLINE 20\%; BVS/LILACS e no Google acadêmico 4\%); ganho de peso (PubMed/MEDLINE 16\%; BVS/LILACS 4\%); migração do implante de subdérmico (PubMed/MEDLINE 28\%); Influência na função sexual (PubMed/MEDLINE 6\%); outros (PubMed/MEDLINE 28\%; BVS/LILACS 14\%). Na Figura 7, esses índices podem ser identificados, permitindo ainda discriminar quais os principais problemas de saúde comum entre os bancos de dados. 
Research, Society and Development, v. 10, n. 15, e466101523281, 2021

(CC BY 4.0) | ISSN 2525-3409 | DOI: http://dx.doi.org/10.33448/rsd-v10i15.23281

Figura 7. Principais problemas de saúde em comuns detectados nas bases de dados de dados.

\begin{tabular}{|c|c|c|c|c|}
\hline \multicolumn{3}{|c|}{$\begin{array}{l}\text { Tipos de impactos negativos registrados na base de dados no periodo } \\
\qquad(2017-2021)\end{array}$} & $\begin{array}{l}\text { freq. } p / \text { cada } \\
\text { plataforma }(\%)\end{array}$ & $\begin{array}{l}\text { freq. } p / \text { todas as } \\
\text { plataforma }(\%)\end{array}$ \\
\hline \multirow{4}{*}{ ScIELO } & $\begin{array}{l}\text { EXPULSÃO DO } \\
\text { DISPOSITIVO }\end{array}$ & 1 ARTIGO & $50 \%$ & $2 \%$ \\
\hline & $\begin{array}{l}\text { PSEUDO } \\
\text { ANEURISMA }\end{array}$ & 1 ARTIGO & $50 \%$ & $2 \%$ \\
\hline & SANGRAMENTO & 2 ARTIGOS & $50 \%$ & $4 \%$ \\
\hline & $\begin{array}{l}\text { GANHO DE } \\
\text { PESO }\end{array}$ & 1 ARTIGO & $25 \%$ & $2 \%$ \\
\hline \multirow{5}{*}{$\begin{array}{l}\text { Google } \\
\text { acadêmico }\end{array}$} & $\begin{array}{l}\text { EXPULSÃO DO } \\
\text { DISPOSITIVO }\end{array}$ & 1. ARTIGO & $25 \%$ & $2 \%$ \\
\hline & AMENORRÉIA & 1 ARTIGO & $25 \%$ & $2 \%$ \\
\hline & SANGRAMENTO & 10 ARTIGOS & $20 \%$ & $20 \%$ \\
\hline & $\begin{array}{c}\text { GANHO DE } \\
\text { PESO }\end{array}$ & 8 ARTIGOS & $20 \%$ & $16 \%$ \\
\hline & AMENORRÉIA & 2 ARTIGOS & $5 \%$ & $4 \%$ \\
\hline \multirow[t]{6}{*}{ PubMed } & $\begin{array}{l}\text { MIGRAÇÃO DE } \\
\text { IMPLANTE }\end{array}$ & 14 ARTIGOS & $35 \%$ & $28 \%$ \\
\hline & ACNE & 1 ARTIGO & $2,5 \%$ & $2 \%$ \\
\hline & $\begin{array}{l}\text { INFLUÉNCIA NA } \\
\text { FUNÇÃO SEXUAL }\end{array}$ & 3 ARTIGOS & $7,5 \%$ & $6 \%$ \\
\hline & OUTROS & 14 ARTIGOS & $35 \%$ & $28 \%$ \\
\hline & SANGRAMENTO & 2 ARTIGOS & $50 \%$ & $4 \%$ \\
\hline & $\begin{array}{l}\text { GANHO DE } \\
\text { PESO }\end{array}$ & 2 ARTIGOS & $50 \%$ & $4 \%$ \\
\hline \multirow[t]{3}{*}{ LILACS } & $\begin{array}{l}\text { EXPULSÃO DO } \\
\text { DISPOSITIVO }\end{array}$ & 1 ARTIGO & $25 \%$ & $2 \%$ \\
\hline & AMENORRÉIA & 1 ARTIGO & $25 \%$ & $2 \%$ \\
\hline & OUTROS & 7 ARTIGO & $175 \%$ & $14 \%$ \\
\hline
\end{tabular}

Fonte: Autores (2021). 
De acordo com os dados apresentados na Figura 7, os principais problemas de saúde identificados levando-se em consideração a relevância das plataformas foram: (i) Maior incidência na plataforma com maior relevância estatística para a busca: PubMed (Migração de implante: 28\%; sangramento: 20\%; ganho de peso: 16\%; influência sexual: 6\%; LILACS (Sangramento: 4\%; ganho de peso: 4\%); Google acadêmico (Sangramento: 4\%). Não foram encontrados problemas de saúde com incidência igual ou maior, pertencentes a base SciELO em comum às outras bases. Os valores de incidência relativos a "outros" não foram levados em consideração para as 04 bases de dados, em virtude de não serem importantes no presente estudo, isto é, incidência estatística baixa e não comum entre as bases de dados. No gráfico representado na Figura 8, esse fato pode ser melhor observado.

Figura 8. Principais problemas de saúde causados pelo uso dos dispositivos contraceptivos reversíveis de longa duração em cada base de dados.

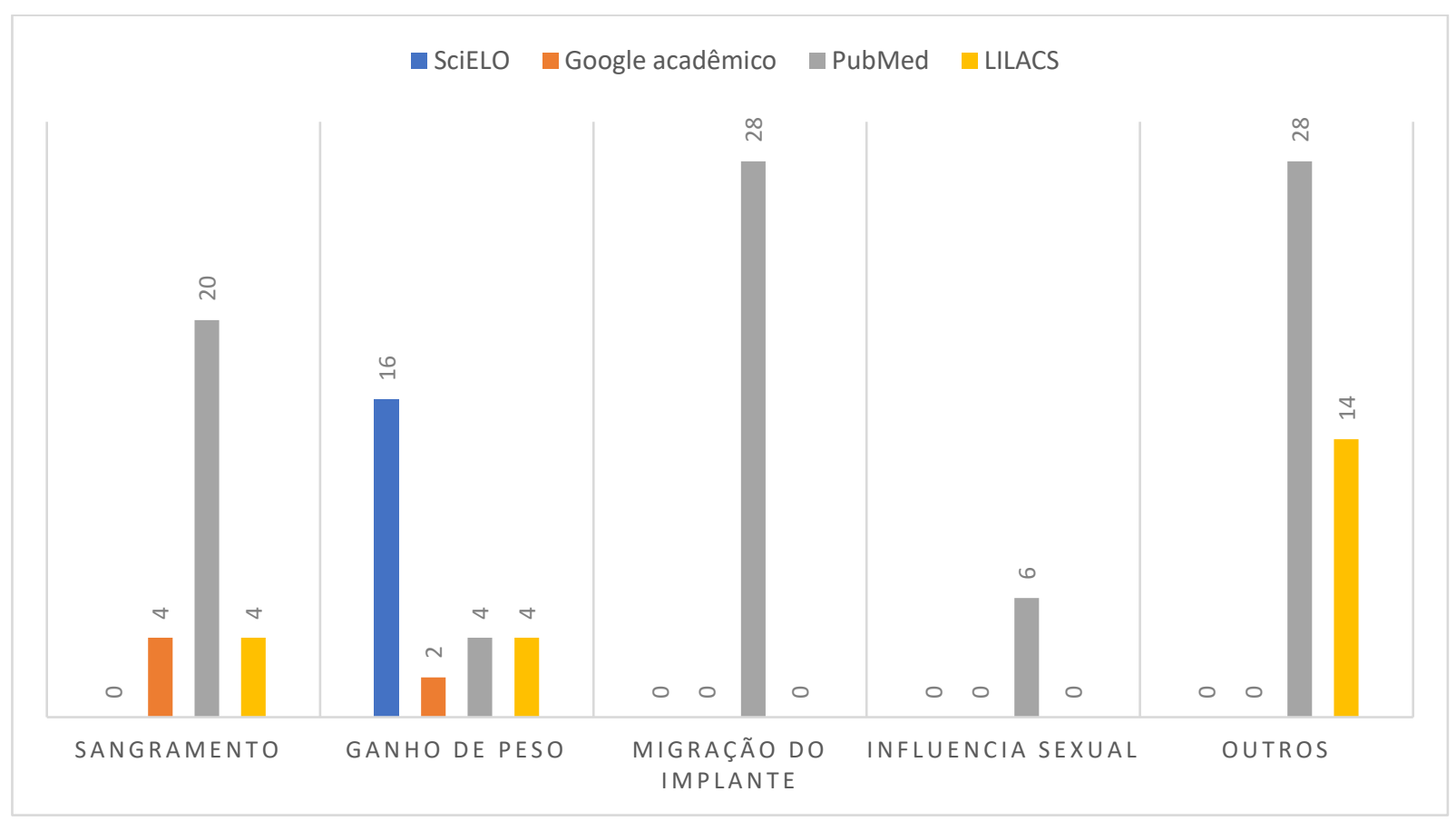

Fonte: Autores (2021).

Ainda, foram identificadas desvantagens e vantagens associadas ao uso de dispositivos contraceptivos de longa duração. Com relação às desvantagens, observou-se que os resultados relativos a sangramentos provocados em mulheres que usaram dispositivos intrauterinos, foram similares aos relatados em outros trabalhos. Em Slywitch et al. (2021), mostrou-se que entre 72 mulheres que utilizaram dispositivos intrauterinos, e que trabalhavam no manuseio de aparelhos de ressonância magnética, 68 apresentaram sangramento abdominal anormal; na revisão sistemática realizada em Ti et al. (2020), foi verificado que o uso de dispositivo intrauterino provocou sangramentos e dores abdominais em 04 entre cada 100 mulheres, acarretando assim a descontinuidade no uso desses dispositivos; já em Sanders et al. (2018), foram observados sangramentos causados pelo uso de dispositivos intrauterinos de cobre, logo após as suas inserções nos úteros das mulheres. Quanto ao ganho de peso feminino, outros trabalhos também apresentaram resultados semelhantes, como em Luz, Barros \& Branco (2021); Fierro-Calpa (2021) e Trindade, Siqueira, Paula \& Mendes (2021), que demonstraram que uso dos dispositivos intrauterinos hormonais apresentaram como um dos efeitos adversos, ganho de peso em mulheres. Em relação à migração ou expulsão do dispositivo, autores como Adeyemi-Fowode \& Bercaw-Pratt (2019), identificaram através de estudo comparativo (coorte), que por meio de uma amostra contendo 281 adolescentes e 571 mulheres entre 20 a 30 anos, permitiram a eles concluir que a mulheres (e/ou adolescentes) que 
utilizaram o dispositivo intrauterino de cobre, apresentaram maiores índices de dores, sangramento, expulsão e deslocamento do dispositivo comparadas as mulheres que usaram os dispositivos intrauterino hormonal.

Com relação às vantagens, outros trabalhos relataram que o uso de dispositivos intrauterinos apresenta mais benefícios em contrapartida dos problemas de saúde que eles causam à saúde feminina. Autores como Machado (2017), mostraram que os benefícios superam os malefícios causados à saúde feminina; em Hofmeyr, Singata \& Lawrie (2010), foi mostrado que o risco das mulheres usuárias de dispositivos intrauterinos desenvolverem doença pélvica inflamatória é bastante baixo quando comparado a outros métodos contraceptivos. Além disso, foi observado que na ocorrência de alguma infecção uterina em mulheres usuárias de dispositivos intrauterinos, o tratamento de combate à infecção responde de forma mais rápida com a retirada dos dispositivos Jatlaoui et al. (2017). Outra vantagem no uso de dispositivos intrauterinos é observada no tratamento de fluxo menstrual intenso, agindo como uma ferramenta de suspensão da menstruação (Malmborg, Brynhildsen \& Hammar, 2019).

Em resumo, de acordo com que foi exposto acima, a utilização dos dispositivos intrauterinos não se resume à prática contraceptiva. Apesar de causarem alguns problemas de saúde em um número pequeno de mulheres, seus benefícios quando comparados a esses problemas são bem menores.

\section{Considerações Finais}

De acordo com os resultados obtidos foi possível identificar quais os principais problemas de saúde associados ao uso de dispositivos contraceptivos reversíveis de longa duração. Também se identificou quais bases de dados é possível recorrer quando se pretende fazer uma revisão sistemática com um intervalo de tempo curto (período de 05 anos), desta forma foi possível observar que o presente trabalho cumpriu com os objetivos propostos, no início da pesquisa.

O trabalho apresenta limitações relativas a utilizar apenas 04 bases de dados. Outra limitação é com relação ao não uso de documentos pertencentes à chamada literatura cinza, ou seja, resumos de artigos em anais de congressos, conferências, simpósio etc. Isso pode representar um pequeno viés associado à utilização de apenas artigos de periódicos.

As contribuições desta pesquisa permitem fazer revisão da literatura referente aos problemas de saúde associados ao tema desta pesquisa relativamente de forma rápida reduzindo a quantidade de bases de dados, consequentemente a quantidade de artigos sem comprometer o resultado obtido.

Verificou-se também que os principais problemas de saúde relatados foram sangramento, ganho de peso e a migração do implante foi incluída devido ao percentual elevado na plataforma PubMed/MEDLINE.do implante causados pelo uso dispositivos contraceptivos reversíveis de longa duração. Além de enfatizar a importância de mais pesquisas dentro dessa área de atuação para maiores contribuições clínicas na conduta da saúde feminina.

\section{Agradecimentos}

Os autores agradecem as seguintes instituições: Universidade de Aveiro - PT, ao Instituto de Telecomunicações de Aveiro - PT, Instituto Federal do Maranhão - BR e a Universidade Nilton Lins - BR, pelo apoio técnico-financeiro prestado.

\section{Referências}

Adeyemi-Fowode, O. A. A., \& Bercaw-Pratt, J. L. (2019). Intrauterine devices: effective contraception with noncontraceptive benefits for adolescents. Journal of pediatric and adolescent gynecology, 32(5), S2-S6. DOI: 10.1016/j.jpag.2019.07.001

Albuquerque, J. S. (2018). Métodos anticoncepcionais reversíveis: uma revisão (Trabalho de Conclusão de Curso). Universidade de Campina Grande, 50.

Bahamondes, L \& Monteiro, I. (2017). Comments on manuscript: vaginal misoprostol prior to intrauterine device insertion in women delivered only by elective cesarean section: a randomized double-blind clinical trial. Contraception, 95(4), 434-435. DOI: 10.1016/j.contraception.2017.01.012

Bassis, C. M., Allsworth, J.E.,Wahl, H.N., Sack, D.E., Young, V.B. \& Bell, J.D. (2017). Effects of intrauterine contraception on the vaginal microbiota. Contraception, 96(3), 189-195. DOI: $10.1016 /$ j.contraception.2017.05.017 
Bearak, J., Popinchalk, A., Alkema, L., \& Sedgh, G. (2018). Global, regional, and subregional trends in unintended pregnancy and its outcomes from 1990 to 2014: estimates from a Bayesian hierarchical model. The Lancet Global Health, 6(4), e380-e389. DOI: 10.1016/S2214-109X(18)30029-9

Boisen, A.B., Ostenfeldt, E.B., Erikstrup, L.T., \& Bay, B. (2017). Genital actinomycosis and pelvic abscesses in a woman with a 13-year-old intrauterine device. UgeskrLaeger, 179(13).

Brandão, E. R. (2019). Métodos contraceptivos reversíveis de longa duração no Sistema Único de Saúde: o debate sobre a (in) disciplina da mulher. Ciência e Saúde Coletiva, 24(3). https://doi.org/10.1590/1413-81232018243.10932017

Carvalho, N.M., Chou, V., Modesto, W., Margatho, D., Garcia, E.A.L. \& Bahamondes, L. (2017). Relationship between user satisfaction with the levonorgestrelreleasing intrauterine system and bleeding pattern. The Journal of Obstetrics and Gyneacology Research, 43(11), 1732-1737. DOI: 10.1111 / jog.13441

Clancy, A.A., Gerritze, R. \& Pascali, D. Intrauterine device visualized as extrinsic bladder mass on citoscopy. (2017). International Urogynecology Journal, 28(9), 1429-1430. DOI: 10.1007/s00192-017-3382-8

Chai, W., Zhang, W., Jia, G., Cui, M. \& Cui, L. (2017). Vesical transmigration of an intrauterine contraceptive device: A rare case report and literature review. Medicine (Baltimore), 96(40), 8236. DOI: 10.1097 / MD.0000000000008236

Cim, N., Soysal, S., Sayan, S., Yildizhan, B., Karaman, E., Cetin, O., Tolunay, H.E. \& Yildizhan, R. (2017). Two Years Follow-Up of Patients with Abnormal Uterine Bleeding after Insertion of the Levonorgestrel-Releasing Intrauterine System. Gynecologic and Obstetric Investigation, 83(6), 569-575. DOI: 10.1159/ 000480012

Couto, M. C., Saiani, C. C., \& Kuwahara, M. Y. (2020). Contracepção e Autonomia das Mulheres na Decisão pela Gravidez: Efeitos do Programa Bolsa Família. Anais do $48^{\circ}$ Encontro Nacional de Economia. https://brsa.org.br/wp-content/uploads/wpcf7-submissions/1886/ENABER-2020-PBF-e-NNMC-comidentifica\%C3\%A7\%C3\%A3o-Copia.pdf

Colwill, A.C., Schreiber, C.A., Sammel, M.D. \& Sonalkar, S. (2017). Six-week retention after post-placental copper intrauterine device placement. Contraception, 97(3):215-218. DOI: $10.1016 /$ j.contraception.2017.10.012

Cortessis, V.K., Barrett, M., Wade, N.B., Enebish, T., Perrigo, J.L., Tobin, J., Zhong, C., Zink, J., Isiaka, V., Muderspach, L.I., Natavio, M. \& McKean-Cowdin, R. (2017). Intrauterine Device Use and Cervical Cancer Risk: A Systematic Review and Meta-analysis. Obstetrics Gynecolology, 130(6), 1226-1236 DOI: 10.1097 / AOG.0000000000002307

Darney, P.D., Stuart, G.S., Thomas, M.A., Cwiak, C., Olariu, A. \& Creinin, M.D. (2017). Amenorrhea rates and predictors during 1 year of levonorgestrel 52 $\mathrm{mg}$ intrauterine system use. Contraception, 97(3):210-214. DOI: 10.1016/ j.contraception.2017.10.005

Dinehart E., Lathi, E.B. \& Aghajanova, L. (2020). Levonorgestrel IUD: is there a long-lasting effect on return to fertility? Journal of Assisted Reproduction and Genetics. 37: 45-52. DOI: 10.1007/s10815-019-01624-5

Donders, G.G.G., Bellen, G., Ruban, K.. \& Bulck, B. V. (2018). Short-and long-term influence of the levonorgestrel-releasing intrauterine system (Mirena) on vaginal microbiota and Candida. Journal of Medical Microbiology. 67(3), 308-313. DOI: 10.1099 / jmm.0.000657

Enfermagem Florence. (2020). Métodos Contraceptivos: Tipos e Eficácia. https://enfermagemflorence.com.br/metodos-contraceptivos-tipos-e-eficacia/adesivocutaneo/.

Fleith, D. D. S., Gomes, C. M. A., Araújo, C. M.M. \& Almeida, L. S. (2020). Expectativas de Sucesso Profissional de Ingressantes na Educação Superior: Estudo Comparativo. Avaliação Psicológica, 19(3), 223-231. http://dx.doi.org/10.15689/ap.2020.1903.17412.01

Fierro-Calpa, L. R. (2021). Cuidados de enfermería en la mujer adulta. Boletín Informativo CEI, 8(1), 94-98.

Fontenelle, L. (2021). Gravidez com DIU? Entenda por que foto de bebê com dispositivo na mão não deve abalar eficácia do método. https://www.oitomeia.com.br/noticias/saude/2021/07/08/gravidez-com-diu-entenda-por-que-foto-de-bebe-com-dispositivo-na-mao-nao-deve-abalar-eficaciado-metodo/.

Grandi, G; Farulla, A; Sileo, F. G \& Facchinetti, F. (2018). Levonorgestrel-releasing Intra-uterine System as Female Contraceptives. Expert Opin Pharmacother. (19)7, 677-686. DOI: 10.1080/14656566.2018.1462337

Giordano, M.V., Giordano, L.A. \& Panisset, K. S. (2015). Dispositivo intrauterino de cobre. FEMINA, 43(1). http://files.bvs.br/upload/S/01007254/2015/v43nsupp11/a4850.pdf

Hofmeyr G. J., Singata M. \& Lawrie, T. (2010). Copper containing intra-uterine devices versus depot progestogens for contraception. Cochrane Database of Systematic Reviews, (6), 1-23.DOI: 10.1002/14651858.CD007043.pub2.

Huss, A., Schaap, K. \& Kromhout, H. (2017). A survey on abnormal uterine bleeding among radiographers with frequente MRI exposure using intrauterine contraceptive devices. Magnetic Resonance in Medicine, 79(2), 1083-1089. DOI: 10.1002/mrm.26707

Inal, Z.O., Inal, H.A. \& Gorkem, U. (2017). Experience of Tubo-Ovarian Abcess: A Retrospective Analysis of 318 Patients in a Single Tertiary Center in Middle Turkey. Surgical Infections (Larchmt), 19(1), 54-60. DOI: 10.1089/sur.2017.215

Jatlaoui, T. C., Riley, H. E., \& Curtis, K. M. (2017). The safety of intrauterine devices among young women: a systematic review. Contraception, 95(1), 17-39. DOI: $10.1016 /$ j.contraception.2016.10.006 
Junges, A. P. P., Andrade, A. M. D., Silva, J. M., Brandão, M. G., De Jorge, V., Maroso, G. S., \& Lubianca, J. N. (2021). Métodos contraceptivos reversíveis de

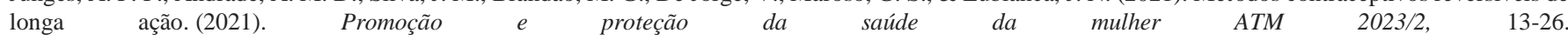
https://lume.ufrgs.br/bitstream/handle/10183/223064/001127622.pdf?sequence=1\&isAllowed=y

Lullo, J.J., Ethington, E., Arshanapalli, A., Reserva, J., Jiang, A., Adams, W., Graziano, S. \& Tung, R. (2017). Incidence of androgenic dermatological side effects following placement of a levonorgestrel intrauterine device of menorrhagia: A survey-based study. Journal of American Academy of Dermatology, 79(2), 364-365. DOI: $10.1016 /$ j.jaad.2017.12.051

Luz, A. L. R., Barros, L. D. S. R., \& Branco, A. C. D. S. C. (2021). Métodos contraceptivos: Principais riscos e efeitos adversos. Revista de Casos e Consultoria, 12(1), e24112-e24112.

Fontenelle, L. (2021). Gravidez com DIU? Entenda por que foto de bebê com dispositivo na mão não deve abalar eficária do método. https://www.oitomeia.com.br/noticias/saude/2021/07/08/gravidez-com-diu-entenda-por-que-foto-de-bebe-com-dispositivo-na-mao-nao-deve-abalar-eficaciado-metodo/.

Machado, R. B. (2017). Uso de dispositivos intrauterinos (DIU) em nulíparas - São Paulo: Federação Brasileira das Associações de Ginecologia e Obstetrícia (FEBRASGO)

Malmborg, A., Brynhildsen, J., \& Hammar, M. (2019). A survey of young women's perceptions of the influence of the Levonorgestrel-Intrauterine System or copper-intrauterine device on sexual desire. Sexual \& Reproductive Healthcare, 21, 75-80.

Madliki, M.S., Hofmeyr, G.J., d'Hellencourt, F.C.L. \& Lawrie, T.A. (2017). Phychological, behavioural and physiological effects of three long-acting reversible contraception (LARC) methods: protocol for an ancillary study of the ECHO randomised trial. Britisg Medical Journal Open (BMJ), 7(11):e019205. DOI: 10.1136 / bmjopen-2017-019205

Mendes, L. V. (2021). Satisfação com o uso do implante contraceptivo e do dispositivo intrauterino com cobre entre usuárias de um hospital público da cidade de São Paulo [Dissertação de Doutorado]. Universidade de São Paulo.

Milletler, B. (2020). The world's women 2020: Trends and statistics. Erişimadresi: https://worldswomen-2020-data-undesa. hub. arcgis. com/pages/violenceagainst-women-and-the-girl-child.

Mørch, L.S., Skovlund, C.W., Hannaford, P.C., Iversen, L., Fielding, S. \& Lidegaard, Ø. (2017). Contemporary Hormonal Contraception and the Risk of Breast Cancer. The New England Journal of Medicine, 377(23), 2228-2239. DOI: 10.1056/NEJMoa1700732

Nakashira, E.S., Maximiano, L.F., Lima, F.R. \& Ussami, E.Y. (2017). Abdominal and pelvic actinomycosis due to longstanding intrauterine device: a slow and devasting infection. Autopsy \& Case Report, 7(1):43-47. DOI: 10.4322 / acr.2017.001

O’Brien, P.A. \& Pillai, S. (2017). Uterine Perforation by intrauterine devices: a 16-year review. The Journal of Family Planning and Reproductive Health Care, 43(4):289-295. DOI: 10.1136 / jfprhc-2016-101684

Parlakgumus, A., Parlakgumus, H.A. \& Ezer, A. (2017). A Rare Case of Acute Appendicitis: Migration of an Intrauterine Device. Journal of the College of Physicians Surgeons-Parkistan: JCPSP, 27(5), 323-324. DOI: 10.1002 / ccr3.4283

Paul, J., \& Criado, A. R. (2020). The art of writing literature review: What do we know and what do we need to know? International Business Review, 29(4), https://doi.org/10.1016/j.ibusrev.2020.101717

Rodrigues, E. W., Jr., Rodrigues, A. C., Silva, I. A. R., \& Ferreira, G. M. (2021). A INSERÇÃO DA MULHER NO MERCADO DE TRABALHO NA ÁREA DA TECNOLOGIA. Revista Eletrônica da Faculdade Invest de Ciências e Tecnologia, $3(1)$. http://revista.institutoinvest.edu.br/index.php/revistainvest/article/view/32/25

Rose, S.B., Garrett, S.M., Stanley, J. \& Pullon, S.R.H. (2017). Chlamydia testing and diagnosis following initiation of long-acting reversible contraception: A retrospective cohort study. Australian and New Zealand Journal of Obstetrics and Gyneacology, 57(6), 665-675. DOI: 10.1111 / ajo.12685

Sanders, J. N., Turok, D. K., Gawron, L. M., Law, A., Wen, L., \& Lynen, R. (2017). Two-year continuation of intrauterine devices and contraceptive implants in a mixed-payer setting: a retrospective review. American journal of obstetrics and gynecology, 216(6), 590-e1. https://doi.org/10.1016/j.ajog.2017.02.003

Sano, M.., Nemoto, K., Miura, T. \& Suzuki, Y. (2017). Endoscopic Treatment of Intrauterine Device Migration into the Bladder with Stone Formation. The Journal of Endourology Case Report, 3(1):105-107. DOI: 10.1089 / cren.2017.0038

Santos, L. J. M. L. (2020). Mulheres no mercado de trabalho: um estudo com mulheres em cargos de liderança (Trabalho de Conclusão de Curso). Centro Universitário Fametro, 29.

http://repositorio.unifametro.edu.br/jspui/bitstream/123456789/532/1/LIZ\%20JORDELLE\%20MOURA\%20LIMA\%20DOS\%20SANTOS_TCC.pdf

Santos, O., Rigo, G.V., Macedo, A.J. \& Tasca, T. (2017). Trichonomas vaginalis clinical isolates: cytodherence and adherence to polystyrene, intrauterine device, and vaginal ring. Parasitology Research, 116(12), 3275-3284. DOI: 10.1007 / s00436-017-5638-0

Sharma, A., Andankar, M. \& Pathak, H. (2017). Intravesical Migration of an intrauterine Contraceptive Device with Secondary Calculus Formation. Korean Journal of Family Medicine, 38(3):163-165. DOI: 10.4082 / kjfm.2017.38.3.163

Silva, C. L. A., Angelo, L. K. G., Bernadino, A. C., Silva, C. A. A., Candido, S. A., Pacheco, A. L. D., Melo, I. S. \& Castro, O. W. (2021). Importância da escola no conhecimento empírico sobre infecções sexualmente transmissíveis e métodos contraceptivos: promoção da saúde, na rede pública de ensino. Brazilian Journal of Development. 7(2), 20421-20432. DOI:10.34117/bjdv7n2-606 
Silva, S.B., Ferreira, T.L.R.L., Fernandes, C., Nogueira, D., Barros, E.J., Mota, R., Oliveira, V.C.C. \& Mendonça, B. (2014). Conhecimentos sobre métodos contraceptivos de acadêmicos de enfermagem da faculdade Montes Belos, em São Luís de Montes Belos-GO. Revista Faculdade Montes Belos (FMB), 8(4), $143-202$.

Silva, W.S.L., Kodithuwakku, K.A.S.U.A., Aponsu, G.U.E., Rathnayake, R.M.M. \& Rajasegaram, E. (2017). A large bladder stone caused by the intravesical migration of an intrauterine contraceptive device: a case report. Jornal of Medical Case Report, 11(1):293. DOI: 10.1186/s13256-017-1461-6

Slywitch, N. C., Alves, B. P., de Paula Martins, E. A., Romão, J. V., Amorim, M. S., Vilela, M. P. D. \& Novais, D. F. F. (2021). Comparação entre os dispositivos intrauterinos de cobre e hormonal: uma revisão narrativa. Revista Eletrônica Acervo Saúde, 13(5), e7345-e7345.

Simon, D.A., Smith, A.L. \& Holzhauer, J.R. (2017). Case Report of an Ectopic Molar Pregnancy in the Presence of an Intrauterine Device. WMJ: official publication of the State of the State Medical Society of Wisconsin, 116(4): 215-217.

Ti, A. J., Roe, A. H., Whitehouse, K. C., Smith, R. A., Gaffield, M. E., \& Curtis, K. M. (2020). Effectiveness and safety of extending intrauterine device duration: a systematic review. American journal of obstetrics and gynecology, 223(1), 24-35. DOI: 10.1016/j.ajog.2020.01.014.

Toumi, O., Ammar, H., Ghdira, A., Chhaidar, A., Trimech, W., Gupta, R., Salem, R., Saad, J., Korbi, I., Nasr, M., Noomen, F., Golli, M. \& Zouari, K. (2017). Pelvic abcess complicating sigmoid colon perforation by migrating intrauterine device: A case report and review of the literature. International Journal of Surgery Case Report, 42:60-63. DOI: 10.1016 / j.ijscr.2017.10.038

Trindade, R. E. D., Siqueira, B. B., Paula, T. F. D., \& Mendes, M. S. F. (2021). Uso de contracepção e desigualdades do planejamento reprodutivo das mulheres brasileiras. Ciência \& Saúde Coletiva, 26, 3493-3504. https://doi.org/10.1590/1413-81232021269.2.24332019

Uçar, M.G., Şanlıkan, F., Ilhan, T.T., Göçmen, A. \& Çelik, C. (2017). Management of intra-abdominally translocated contraceptive devices, is surgery the only way to treat this problem?. Journal of Obstetric Gynaecology, 37(4):480-486. DOI: 10.1080 / 01443615.2016.1268577

Vasconcellos, E. D. C. C. \& Brisolla, S. N. (2016). Presença feminina no estudo e no trabalho da ciência na Unicamp. Cadernos Pagu, 32:215-265.

Visentini, A. P. (2021). Mulheres em transição de carreira: significados para a saída de carreiras corporativas. (Dissertação de Mestrado). Universidade Federal do Rio Grande do Sul, 139.

Zhao, X., Liu, Q., Sun, H., Hu, Y. \& Wang, Z. (2017). Chronic Systemic Toxicity Study of Copper Intrauterine Devices in Female Wistar Rats. Medical Science Monitor, 23:3961-3970. DOI: 10.12659/msm.902137 